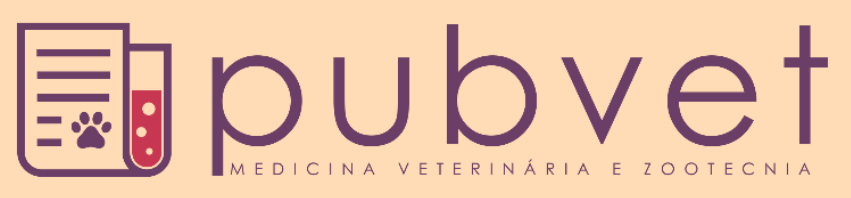

https://doi.org/10.31533/pubvet.v13n10a439.1-10

\title{
Glicerina bruta ou glicerol na dieta de cordeiros: Uma metanálise - desempenho, carcaça e carne
}

\author{
Gustavo Daniel Vega Britez ${ }^{\bullet}$, Ariádne Patrícia Leonardo ${ }^{2 *} \bullet$, José Augusto Velazquez Duarte ${ }^{\bullet}$, Nelson \\ David Lesmo Duarte $^{1 \bullet}$, Marcos Arturo Ferreira Aguero $^{1}{ }^{\circ}$, Fernando Miranda de Vargas Junior ${ }^{\circ}{ }^{\circ}$ \\ I Professor da Universidad Nacional de Asunción, Departamento de Produccion Animal, Pedro Juan Caballero, Paraguay. \\ ${ }^{2}$ Pesquisadora colaboradora da Universidade Federal da Grande Dourados, Departamento de Zootecnia, Dourados-MS, Brasil. \\ ${ }^{3}$ Professor da Universidade Federal da Grande Dourados, Departamento de Ciências Agrárias, Dourados-MS, Brasil. \\ *Autor para correspondência, E-mail: aripatiileonardo@hotmail.com
}

Resumo. A intensificação da produção de biodiesel gera inevitavelmente o subproduto glicerina bruta, que tem pouca saída para outros usos principalmente por sua pureza. A inclusão de glicerina bruta (GB) ou glicerol (G) como fonte de energia, principalmente como substituto do milho, pode ser uma alternativa viável no acabamento de cordeiros. Nesse sentido, objetivaram-se avaliar os níveis de GB e G na dieta sobre os desempenhos, características da carcaça e qualidade de carne de cordeiros através de um estudo metanalítico. Foram selecionados 24 artigos de alto impacto com pesquisas realizadas no Brasil e no exterior, envolvendo 880 cordeiros distribuídos em 86 tratamentos. Os dados, tanto de GB e $\mathrm{G}$ foram agrupados em níveis. Com base nos dados contidos nestes artigos foram analisados: o peso vivo inicial (PVI), peso vivo ao abate (PVA), consumo de massa seca (CMS), ganho médio diário (GMD), conversão alimentar (CA), rendimento de carcaça quente (RCQ), rendimento de carcaça fria (RCF), área de olho de lombo (AOL), espessura de gordura (EG), ácidos graxos saturados (AGS), insaturados (AGI), monoinsaturados (AGMI), poli-insaturados (AGPI), relação n6:n3, proteína bruta (PB), extrato etéreo (EE), $\mathrm{pH}$ final e força de cisalhamento em relação ao tratamento controle $(0 \%$ de GB ou G na MS). Os dados obtidos foram submetidos à análise de variância para efeito linear e quadrático em cada uma das variáveis dentro de todos os níveis e, no caso de significância, foi realizada análise de regressão. O desempenho, características da carcaça e qualidade da carne de cordeiros até 36,0\% de inclusão na MS da dieta de cordeiros de GB e 32,4\% de G não causaram efeito deletério nas mesmas, podendo enquadrar-se como seguro para consumo humano. GB e $\mathrm{G}$ apresentam-se como alternativa na dieta de cordeiros em acabamento, porém, a estratégia do uso como substituto energético deve vir em conjunto com o estudo da viabilidade econômica com base no preço do milho, glicerina bruta e demanda de cordeiros acabados na região em questão.

Palavras chaves: biodiesel, consumo, qualidade da carne, ruminantes, subproduto

\section{Crude glycerin or glycerol in the lamb diet: A meta-analysis - performance, carcass and meat}

Abstract. The intensification of biodiesel production inevitably generates the crude
glycerine byproduct, which has little output for other uses mainly due to its purity. The
inclusion of crude glycerin (GB) or glycerol (G) as an energy source, mainly as a corn
substitute, may be a viable alternative in lamb finishing. In this sense, the objective of this
study was to evaluate the levels of GB and G in the diet on lamb performance, carcass
characteristics and meat quality through a metanalytic study. Twenty-four high impact
articles were selected from research conducted in Brazil and abroad, involving 880 lambs 
distributed in 86 treatments. Data from both GB and G were grouped into levels. Based on the data contained in these articles were analyzed: initial live weight (PVI), slaughter live weight (PVA), dry matter intake (CMS), average daily gain (GMD), feed conversion (CA), carcass yield (RCQ), cold carcass yield (RCF), loin eye area (AOL), fat thickness (EG), saturated fatty acids (AGS), unsaturated (AGI), monounsaturated (AGMI), polyunsaturated (AGPI) , n6: $\mathrm{n} 3$ ratio, crude protein (CP), ether extract (EE), final $\mathrm{pH}$ and shear force in relation to control treatment ( $0 \% \mathrm{~GB}$ or $\mathrm{G}$ in $\mathrm{MS})$. The obtained data were submitted to analysis of variance for linear and quadratic effect in each of the variables within all levels and, in the case of significance, regression analysis was performed. The performance, carcass characteristics and meat quality of lambs up to $36.0 \%$ of inclusion in the DM of GB and $32.4 \%$ of lambs diet did not have deleterious effect on them, and can be considered safe for human consumption. GB and $\mathrm{G}$ are presented as an alternative in the diet of finishing lambs, however, the strategy of use as energy substitute should come together with the study of the economic viability based on the price of corn, crude glycerin and demand of finished lambs in the region in question.

Keywords: biodiesel, consumption, meat quality, ruminants, by-product

\section{Glicerina cruda o glicerol em la dieta de corderos: metanálisis - rendimiento de producción, canal y carne}

Resumen. La intensificación de la producción de biodiésel genera inevitablemente el subproducto glicerina cruda, que tiene poca salida para otros usos principalmente por su pureza. La inclusión de glicerina cruda (GB) o glicerol (G) como fuente de energía, principalmente como substituto del maíz, puede ser una alternativa viable en la terminación de corderos. En ese sentido, se tuvo como objetivo evaluar los niveles de GB y G en la dieta sobre los desempeños, características de la canal y calidad de carne de corderos a través de un estudio meta-analítico. Fueron seleccionados 24 artículos de alto impacto con investigaciones realizadas en Brasil y en el exterior, totalizando 880 corderos distribuidos en 86 tratamientos. Los datos, tanto de GB y G fueron agrupados en niveles. Con base en los datos contenidos en estos artículos fueron analizados: el peso vivo inicial (PVI), peso vivo a la faena (PVF), consumo de materia seca (CMS), ganancia promedio diario (GPD), conversión alimentar (CA), rendimiento de canal caliente (RCQ), rendimiento de canal fría (RCF), área de ojo de bife (AOB), espesura de gordura (EG), ácidos grasos saturados (AGS), insaturados (AGI), monoinsaturados (AGMI), poliinsaturados (AGPI), relación n6:n3, proteína bruta $(\mathrm{PB})$, extracto etéreo $(\mathrm{EE}), \mathrm{pH}$ final y fuerza de cizallamiento en relación al tratamiento testigo ( $0 \%$ de GB o $\mathrm{G}$ en la MS). Los datos obtenidos fueron sometidos a análisis de variancia para efecto lineal y cuadrático en cada una de las variables dentro de todos los niveles y, en el caso de significancia, fue realizada análisis de regresión. El desempeño, características de la canal y calidad de la carne de corderos hasta 36,0\% de inclusión en la MS de la dieta de corderos de GB y 32,4\% de G no causaron efecto perjudicial en las mismas, pudiendo encuadrarse como seguro para consumo humano. GB y G se presentan como alternativa en la dieta de corderos en terminación, sin embargo, la estrategia de uso como substituto energético debe ir acompañado de un estudio de viabilidad económica con base en el precio del maíz, glicerina cruda y demanda de corderos terminados en la región en cuestión.

Palabras clave: biodiésel, consumo, calidad de la carne, rumiantes, subproductos

\section{Introdução}

A produção de biodiesel a partir de matérias-primas vegetais ou animais gera um produto secundário, a glicerina. Para cada tonelada desse biodiesel produzido, gera-se até $103 \mathrm{~kg}$ de glicerina bruta (Nobrega et al., 2012; Quispe et al., 2013). Segundo a ANP (2019), as usinas brasileiras fabricaram um total de 5,3 bilhões toneladas de biodiesel no ano de 2018. 
Dependendo da fonte, a concentração de glicerol na glicerina bruta pode ser baixa (63\%), média (85\%) e alta (>99\%) (Schröder \& Südekum, 1999); sendo as indústrias farmacêuticas e cosméticas as que absorvem as de alta pureza. A glicerina bruta com baixa ou média concentração de glicerol tem despertado o interesse como alternativa de fonte energética em substituição aos grãos nas dietas de animais, colaborando ambientalmente ao dar direcionamento a este resíduo da indústria.

Nas últimas décadas, pesquisas foram direcionadas com intuito de verificar o efeito de substituição do milho e outros grãos energéticos por glicerina bruta na dieta de ruminantes (Chanjula, 2018; Chanjula et al., 2015; Chanjula et al., 2016; Cruz et al., 2014; Eiras et al., 2014a; Eiras et al., 2014b; Eiras et al., 2013; Françozo et al., 2013; Prado et al., 2016; Silva et al., 2014; Silva et al., 2017) ou não ruminante (Egea et al., 2016; Garcia et al., 2019; Gonçalves et al., 2015). Mas poucas pesquisas se atentaram a avaliar o efeito do nível de glicerol que compuseram a glicerina bruta sobre os desempenhos e características das carcaças dos cordeiros. Isto acaba dificultando qualquer comparação entre estes estudos, pois a simples informação do teor de glicerina bruta não é informação suficiente devido à grande variação do teor de glicerol e gordura entre as glicerinas brutas utilizadas.

Diante disto, a hipótese principal desta metanálise é que ao avaliar os resultados com base no nível de glicerol na dieta em vez do nível de glicerina bruta; algumas publicações podem apresentar resultados diferentes. Objetivou-se assim avaliar o efeito de concentração crescente de glicerol (\% da MS) na dieta sobre o desempenho, características qualitativas e quantitativas das carcaças de cordeiros.

\section{Material e métodos}

A base de dados foi constituída por artigos científicos oriundos através de uma revisão sistemática obtida no buscador Google Scholar, Pubmed, Science Direct e Scielo. A busca foi nos meses de janeiro até agosto de 2019, visando artigos publicados entre os anos 2010 até 2019, empregando-se as palavras chaves crude glycerin, glycerol, lambs, performance, carcass characteristics, meat quality.

No processo de busca, foram identificados 55 artigos que constituíram a amostra primária do estudo. Logo foi realizada uma análise pormenorizada de cada artigo e verificou-se a necessidade de procederse o descarte de alguns artigos motivados pela ausência de dados sobre peso inicial (10 artigos), mistura de glicerina bruta com outros ingredientes na dieta ( 8 artigos), trabalhos que não apresentaram dados de porcentagem de glicerol (8) e experimentos conduzidos envolvendo animais adultos como ovelhas ou carneiros (5). Os dados foram obtidos diretamente dos trabalhos publicados para todas as variáveis que correspondem a Glicerina Bruta. Os dados para Glicerol foram obtidos transformando os dados apresentados nos artigos (\% de glicerina bruta na MS x porcentagem de glicerol informado em cada artigo).

As variáveis estudadas foram: o peso vivo inicial (PVI), peso vivo ao abate (PVA), consumo de massa seca (CMS), ganho médio diário (GMD), conversão alimentar (CA), rendimento de carcaça quente (RCQ), rendimento de carcaça fria (RCF), área de olho de lombo (AOL), espessura de gordura (EG), ácidos graxos saturados (AGS), insaturados (AGI), monoinsaturados (AGMI), poli-insaturados (AGPI), razão n6:n3, proteína bruta (PB), extrato etéreo (EE), pH final e força de cisalhamento (FC). Assim, foram formados tratamentos de diferentes níveis de glicerina bruta ou glicerol na MS da dieta. Ante a heterogeneidade ou amplitude dos níveis utilizados nas publicações, houve a necessidade de agrupá-los em intervalos, da seguinte forma:

\section{Glicerina Bruta \%:}

Desempenho e características de carcaça (PVI, PVA, CMS, GMD, CA, RCQ, RCF, AOL e EG): 0\%; $2,5-5,0 \% ; 6,0-10,0 \% ; 12,0-15,0 \% ; 18,0-20,0 \%$ e 21,0-36,0\% de glicerina bruta na MS da dieta dos cordeiros em acabamento.

Ácidos graxos (AGS, AGI, AGMI, AGPI e n6:n3): 0\%; 2,5-10,0\%; 14,0-20,0\% e 21,0-30,0\% de glicerina bruta na MS da dieta dos cordeiros em acabamento.

Composição química da carne e instrumental (PB, EE, pH e FC): 0\%; 2,5-10,0\%; 12,0-20,0\% e 21,0$30,0 \%$ de glicerina bruta na MS da dieta dos cordeiros em acabamento. 


\section{Glicerol \%:}

Desempenho e características de carcaça (PVI, PVA, CMS, GMD, CA, RCQ, RCF, AOL e EG): 0\%; 0,98-4,83\%; 5,28-9,66\%; 10,49-14,49\%; 16,38-18,68\% e 20,98-32,40\% de glicerol na MS da dieta dos cordeiros em acabamento.

Ácidos Graxos (AGS, AGI, AGMI, AGPI e n6:n3): 0\%; 0,98-8,39\%; 10,92-18,68\% e 20,90-24,90\% de glicerol na MS da dieta dos cordeiros em acabamento.

Composição química da carne e instrumental (PB, EE, pH e FC): 0\%; 0,98-8,39\%; 10,92-16,78\% e 21,60-32,490\% de glicerol na MS da dieta dos cordeiros em acabamento.

As análises dos ácidos graxos foram realizadas no músculo Longissimus thoracis et lumborum. Análises de variância e de regressão foram realizadas a partir de dados de cada variável dentro de cada nível de glicerina bruta e glicerol na dieta. Nas variáveis e níveis em que houve significância linear ou quadrática foram determinadas as equações de regressão, de acordo com o modelo que melhor se ajustou. As análises de variância e de regressão foram realizadas com auxílio do software estatístico AgroEstat $^{\circledR}$, ao nível de $5 \%$ de probabilidade $(\mathrm{P}<0,05)$.

\section{Resultados e discussão}

Obteve-se uma amostra final com 24 artigos científicos (Tabela 1), dos quais 19 artigos foram conduzidos no Brasil, dois no EEUU, um no Canadá, um na Espanha e um no Egito. No total, 14 artigos foram publicados em revistas fora do Brasil e 10 artigos publicados em revistas brasileiras. Nos 24 artigos, foram envolvidos 880 cordeiros distribuídos em 86 tratamentos. O principal critério de inclusão foi à presença de tratamento controle $(0 \%)$ nos estudos ou artigo avaliados.

Tabela 1. Pesquisas publicadas utilizadas na metanálise, os diferentes percentuais de glicerol observados na glicerina bruta e os respectivos países de origem

\begin{tabular}{|c|c|c|c|}
\hline Autores & \multicolumn{2}{|c|}{ Glicerol, \% Revistas, Volume, Número e páginas ou DOI } & Pais \\
\hline$\overline{\text { Almeida et al. (2018) }}$ & 83,00 & Animal Production Science, 58(7), 1271-1278 & Brasil \\
\hline Andrade et al. (2018). & 80,50 & Small Ruminant Research, 154, p. 41-47 & Brasil \\
\hline Avila-Stagno et al. (2013) & 99,50 & Journal of Animal Science, 91, 829-837 & Canadá \\
\hline Borghi et al. (2016) & 83,90 & Revista Brasileira de Zootecnia, 45(9), 554-562 & Brasil \\
\hline Carvalho et al. (2015) & 83,00 & Meat Science, 110, 285-292 & Brasil \\
\hline Costa et al. (2019) & 80,50 & Asian-Australas Journal Animal Science, DOI: https://doi.org/10.5713/ajas.18.0825 & Brasil \\
\hline Cunha et al. (2016) & 39,30 & Revista Brasileira de Saúde e Produção Animal, 17(4), p. 729-743 & Brasil \\
\hline D'Aurea et al. (2018) & 83,00 & Arquivo Brasileiro de Medicina Veterinaria e Zootecnia, 70(6), 1950-1958 & Brasil \\
\hline Gomes et al. (2011) & 83,15 & Revista Brasileira de Zootecnia, 40(10), 2211-2219, & Brasil \\
\hline Gunn et al. (2010a) & 89,53 & Journal of Animal Science, 88, 1771-1776 & Brasil \\
\hline Gunn et al. (2010b) & 89,53 & The Professional Animal Scientist, 26, 298-306 & EEUU \\
\hline Lage et al. (2010) & 36,20 & Pesquisa Agropecuária Brasileira, v. 45, n. 9, p. 1012-1020 & EEUU \\
\hline Lage et al. (2014) & 36,20 & Meat Science, 96, 108-113 & Brasil \\
\hline Merlim et al. (2015) & 83,90 & Journal of Agricultural Engineering and Biotechnology, 3(2), 79.83 & Brasil \\
\hline Orrico Jr et al. (2015) & 39,30 & Bioscience Journal, 31, (4), 1152-1158 & Brasil \\
\hline Pellegrin et al. (2012) & 84,80 & Ciência Rural, 42(8), 1477-1482 & Brasil \\
\hline Pellegrin et al. (2013) & 84,80 & Arquivo Brasileiro de Medicina Veterinária e Zootecnia, 65(5), 1509-1518 & Brasil \\
\hline Rego et al. (2017) & 78,00 & Semina: Ciências Agrárias, 38(4), 2051-2064 & Brasil \\
\hline Rezende et al. (2017) & 90,00 & Archives of Animal Nutrition, 71(6), 1-16 & Brasil \\
\hline Romanzini et al. (2018) & 83,90 & Tropical Animal Health and Production, 50(1), 155-160 & Brasil \\
\hline Saleem \& Singer (2018) & 99,00 & Animal, 12(5), 959-963 & Egito \\
\hline Souza et al. (2015) & 88,00 & Small Ruminant Research, 129, 1-5 & Brasil \\
\hline Terré et al. (2011) & 86,20 & Animal Feed Science and Technology, 164, 262-267 & Espanha \\
\hline Vieira \& Silva, et al. (2018) & 80,70 & Revista Brasileira de Zootecnia, 47, p. 1-7 & Brasil \\
\hline Média & 77,75 & & \\
\hline Máximo & 99,50 & & \\
\hline Mínimo & 36,20 & & \\
\hline
\end{tabular}


No total, foram 212 cordeiros da raça Santa Inês (machos não castrados), 102 cordeiros da raça Ripollesa (machos não castrados), 100 cordeiros Ile de France (machos não castrados), 78 cordeiros Suffolk (30 machos não castrados e 48 fêmeas), 64 cordeiros Santa Inês x Dorper (25 machos castrados, 32 machos não castrados), 64 cordeiros que não foram mencionadas as raças (cordeiros lactantes), 60 cordeiros da raça Arcott (machos não castrados), 48 cordeiros Pantaneiro (machos não castrados), 40 cordeiros $1 / 2$ Dorper $1 / 2$ Santa Inês (machos não castrados), 32 cordeiros 1/2 Dorper $1 / 4$ Texel 1/4 Suffolk (machos não castrados), 32 da raça Texel (machos não castrados), 24 cordeiros $1 / 2$ Dorper x Santa Inês (machos não castrados) e 24 da raça Saidi (machos não castrados).

Esta sumarização dos dados permitiu observar a heterogeneidade dos resultados apresentados nas diferentes publicações, onde as maiorias dos autores trabalharam com glicerina bruta, em muitos casos com baixos níveis de glicerol, principalmente aquele desenvolvido no Brasil (Tabela 1), enquanto que pesquisas desenvolvidas fora do Brasil utilizaram glicerina purificada, ou seja, concentração igual ou superior a $90 \%$ de glicerol. Além disso, as pesquisas avaliaram o uso de glicerina bruta na MS em baixas concentrações na dieta. As maiorias das pesquisas avaliaram os desempenhos dos cordeiros e pouco as características qualitativas da carne.

$\mathrm{O}$ fato de que a maioria das pesquisas envolvendo o uso da glicerina bruta na dieta de pequenos ruminantes, neste caso cordeiros, seja desenvolvida no Brasil (Tabela 1) não é casualidade. Isso porque o Brasil é o segundo maior produtor da soja, depois somente do EUA, sendo esta leguminosa a principal fonte de biodiesel no país (César et al., 2019) gerando grande quantidade de subprodutos entre elas a glicerina bruta (Ma \& Hanna, 1999; Quispe et al., 2013). O Brasil é responsável pela produção de 114 milhões de toneladas, pouco abaixo do EUA com 117 milhões toneladas e muito acima de outros países produtores como China (13 milhões toneladas), Índia (12 milhões toneladas) e Paraguai (10 milhões toneladas) (Cattelan \& Dall'Agnol, 2018). Existe a previsão que na safra 2019/2020 o Brasil passe a ser o maior produtor mundial da soja (USDA, 2019).

As respostas dos principais parâmetros de desempenhos de cordeiros em função a diferentes níveis de glicerina bruta e glicerol na dieta sãos apresentados na Tabela 2 e 3 . Não houve efeito $(P>0,05)$ para PVA, CMS, GMD e CA em função aos diferentes níveis de glicerina bruta e glicerol na dieta. Esses resultados indicam que o uso de até $36 \%$ de glicerina bruta na MS (Tabela 2) e até 32,4\% de glicerol na MS (Tabela 3) na dieta dos cordeiros pode ser possível sem acarretar efeito deletério no desempenho dos animais.

Tabela 2. Peso vivo inicial (PVI), peso vivo ao abate (PVA), consumo de massa seca (CMS), ganho médio diário (GMD) e conversão alimentar (CA) em função a diferentes níveis de glicerina bruta na dieta de cordeiros em acabamento

\begin{tabular}{|c|c|c|c|c|c|c|c|c|c|c|}
\hline \multirow{2}{*}{ Variáveis } & \multicolumn{6}{|c|}{ Níveis de Glicerina bruta, \% da MS } & \multirow{2}{*}{ SEM } & \multirow{2}{*}{ Gli } & \multirow{2}{*}{$\mathrm{L}$} & \multirow{2}{*}{ Q } \\
\hline & 0 & $2,5-5,0$ & $6,0-10,0$ & $12,0-15,0$ & $18,0-20,0$ & $21,0-36,0$ & & & & \\
\hline PVI (kg) & 19,56 & 20,01 & 19,24 & 20,77 & 17,05 & 19,62 & 0,512 & 0,551 & 0,062 & 0,858 \\
\hline PVA (kg) & 34,33 & 35,67 & 33,88 & 35,19 & 32,47 & 35,24 & 0,480 & 0,569 & 0,849 & 0,789 \\
\hline CMS (g/dia) & 1136,27 & 1120,62 & 1091,71 & 1092,49 & 1206,93 & 1117,71 & 17,355 & 0,950 & 0,710 & 0,942 \\
\hline GMD (g/dia) & 243,39 & 206,50 & 248,88 & 249,50 & 263,40 & 259,67 & 8,314 & 0,444 & 0,159 & 0,418 \\
\hline $\mathrm{CA}(\mathrm{kg} / \mathrm{kg})$ & 5,33 & 5,67 & 4,67 & 4,69 & 5,15 & 4,84 & 0,163 & 0,757 & 0,331 & 0,518 \\
\hline
\end{tabular}

SEM= erro quadrado da média. Gli= 0 vs. Glicerina. $\mathrm{L}=$ linear. $\mathrm{Q}=$ quadrática.

O CMS variou de 1.136,27 g/dia (0\% glicerina bruta) até 1.117,71 g/dia (21,0\%-36,0\% de glicerina bruta na MS), enquanto, a variação no CMS daqueles cordeiros sem adição de glicerol (0\%) foi de $1.136,27 \mathrm{~g} /$ dia até $1.183,50 \mathrm{~g} /$ dia com a dieta contendo de 20,98\%-32,4\% de glicerina na MS. Esses resultados para CMS sugerem que o glicerol aumenta a eficiência da energia da dieta utilizada pelos microrganismos no rúmen e no animal. Além disso, isso sugere que níveis até $36 \%$ de glicerina bruta e $32,4 \%$ de glicerol não promoveram danos severos ou modificações no ambiente ruminal que prejudicassem o consumo. A não existência de diferenças significativas para os parâmetros avaliados (Tabela 2 e 3) sugerem que a sumarização consegue equiparar melhor os dados, considerando que algumas publicações que obtiveram diferenças significativas no CMS (Almeida et al., 2018; Andrade et al., 2018) e outras não (Avila-Stagno et al., 2013; Rezende et al., 2017; Saleem \& Singer, 2018). 
Tabela 3. Peso vivo inicial (PVI), peso vivo ao abate (PVA), consumo de massa seca (CMS), ganho médio diário (GMD) e conversão alimentar (CA) em função a diferentes níveis de glicerol na dieta de cordeiros em acabamento

\begin{tabular}{|c|c|c|c|c|c|c|c|c|c|c|}
\hline \multirow{2}{*}{ Variáveis } & \multicolumn{6}{|c|}{ Níveis de Glicerol, \% da MS } & \multirow{2}{*}{ SEM } & \multirow{2}{*}{ Gli } & \multirow{2}{*}{$\mathrm{L}$} & \multirow{2}{*}{ Q } \\
\hline & 0 & $0,98-4,83$ & $5,28-9,66$ & $10,49-14,49$ & $16,38-18,68$ & $20,98-32,40$ & & & & \\
\hline PVI (kg) & 19,56 & 20,13 & 18,87 & 21,05 & 16,61 & 19,60 & 0,615 & 0,317 & 0,638 & 0,851 \\
\hline PVA $(\mathrm{kg})$ & 34,33 & 34,45 & 33,91 & 36,51 & 32,37 & 34,98 & 0,553 & 0,439 & 0,977 & 0,994 \\
\hline CMS (g/dia) & 1136,27 & 1031,02 & 1180,52 & 1162,35 & 1041,25 & 1183,50 & 28,188 & 0,371 & 0,671 & 0,902 \\
\hline GMD (g/dia) & 243,39 & 215,24 & 260,49 & 258,70 & 275,29 & 246,83 & 8,342 & 0,156 & 0,360 & 0,352 \\
\hline $\mathrm{CA}(\mathrm{kg} / \mathrm{kg})$ & 5,33 & 4,91 & 5,12 & 4,88 & 4,58 & 5,13 & 0,105 & 0,973 & 0,501 & 0,281 \\
\hline
\end{tabular}

$\mathrm{SEM}=$ erro quadrado da média. Gli= 0 vs. Glicerina. $\mathrm{L}=$ linear. $\mathrm{Q}=$ quadrática.

Trabalhos com baixa concentração de glicerol na glicerina bruta obtiveram diferenças significativas no CMS, diferente dos que informaram alta pureza ou concentração igual ou superior a $90 \%$ de glicerol na glicerina bruta. Provavelmente, os trabalhos com baixo nível de glicerol contenham compostos antinutricionais elevados como metanol, ou outras substâncias, que a maioria dos trabalhos não mencionou.

Tabela 4. Rendimento de carcaça quente (RCQ \%), rendimento de carcaça fria (RCF \%), área de olho de lombo $\left(\mathrm{AOL} \mathrm{cm}^{2}\right) \mathrm{e}$ espessura de gordura (mm) em função a diferentes níveis de glicerina bruta na dieta de cordeiros em acabamento

\begin{tabular}{|c|c|c|c|c|c|c|c|c|c|c|}
\hline \multirow{2}{*}{ Variáveis } & \multicolumn{6}{|c|}{ Níveis de Glicerina bruta, \% da MS } & \multirow{2}{*}{ SEM } & \multirow{2}{*}{ Gli } & \multirow{2}{*}{$\mathrm{L}$} & \multirow{2}{*}{ Q } \\
\hline & 0 & $2,5-5,00$ & $6,00-10,00$ & $12,00-15,00$ & $18,00-20,00$ & $21,00-36,00$ & & & & \\
\hline $\operatorname{RCQ}(\%)$ & 50,56 & 51,43 & 50,63 & 51,15 & 52,05 & 49,17 & 0,401 & 0,769 & 0,465 & 0,229 \\
\hline $\mathrm{RCF}(\%)$ & 47,02 & 47,07 & 46,62 & 46,05 & 46,96 & 46,80 & 0,155 & 0,878 & 0,698 & 0,526 \\
\hline $\mathrm{AOL}\left(\mathrm{cm}^{2}\right)$ & 13,71 & 15,04 & 13,34 & 14,78 & 14,14 & 14,76 & 0,276 & 0,656 & 0,514 & 0,830 \\
\hline $\mathrm{EG}(\mathrm{mm})$ & 2,04 & 1,86 & 2,09 & 1,80 & 2,38 & 1,98 & 0,083 & 0,920 & 0,697 & 0,890 \\
\hline
\end{tabular}

$\mathrm{SEM}=$ erro quadrado da média. Gli= 0 vs. Glicerina. $\mathrm{L}=$ linear. $\mathrm{Q}=$ quadrática.

Níveis de glicerina bruta e glicerol na MS da dieta não apresentaram efeitos $(\mathrm{P}>0,05)$ sobre as variáveis RCQ, RCF, AOL e EG (Tabela 4 e 5). Assim, inclusão de até 36,0\% de glicerina bruta na dieta de cordeiros é possível sem comprometer o RCQ ( $\mathrm{n}=76), \mathrm{RCF}(\mathrm{n}=81), \mathrm{AOL}(\mathrm{n}=75)$ e EG (n=75) (Tabela 4). Enquanto a inclusão de glicerol na dieta de cordeiros pode ser até $32,40 \%$ sem comprometer o RCQ $(\mathrm{n}=68), \operatorname{RCF}(\mathrm{n}=73), \operatorname{AOL}(\mathrm{n}=67)$ e $\mathrm{EG}(\mathrm{n}=67)$ (Tabela 5).

Tabela 5. Rendimento de carcaça quente (RCQ \%), rendimento de carcaça fria (RCF \%), área de olho de lombo $\left(\mathrm{AOL} \mathrm{cm}^{2}\right) \mathrm{e}$ espessura de gordura (mm) em função a diferentes níveis de glicerol na dieta de cordeiros em acabamento

\begin{tabular}{|c|c|c|c|c|c|c|c|c|c|c|}
\hline \multirow{2}{*}{ Variáveis } & \multicolumn{6}{|c|}{ Níveis de Glicerol, \% da MS } & \multirow{2}{*}{ SEM } & \multirow{2}{*}{ Gli } & \multirow{2}{*}{$\mathrm{L}$} & \multirow{2}{*}{ Q } \\
\hline & 0 & $0,98-4,83$ & $5,03-9,66$ & $10,49-14,49$ & $16,60-18,68$ & $20,90-32,40$ & & & & \\
\hline RCQ (\%) & 50,56 & 51,18 & 50,24 & 51,25 & 51,66 & 49,35 & 0,341 & 0,866 & 0,492 & 0,297 \\
\hline $\mathrm{RCF}(\%)$ & 47,02 & 46,11 & 47,06 & 46,75 & 46,89 & 46,83 & 0,141 & 0,817 & 0,718 & 0,944 \\
\hline $\operatorname{AOL}\left(\mathrm{cm}^{2}\right)$ & 13,7 & 13,5 & 13,6 & 15,3 & 15,1 & 14,8 & 0,327 & 0,535 & 0,104 & 0,184 \\
\hline $\mathrm{EG}(\mathrm{mm})$ & 2,04 & 1,70 & 2,35 & 1,94 & 2,63 & 1,99 & 0,135 & 0,414 & 0,600 & 0,620 \\
\hline
\end{tabular}

$\mathrm{SEM}=$ erro quadrado da média. $\mathrm{Gli}=0$ vs. Glicerina. $\mathrm{L}=$ linear. $\mathrm{Q}=$ quadrática.

Enquanto as variáveis qualitativas da carne de cordeiros, o AGMI $(\%)$ foi influenciado $(\mathrm{P}<0,05)$ pelos níveis de glicerina bruta e glicerol na dieta (Tabelas 6 e 7). Assim, concentração de AGS, AGI, AGPI e a razão n6:n3 não foram influenciados $(\mathrm{P}>0,05)$ pelos níveis de glicerina bruta e glicerol nas dietas. À medida que aumenta o nível de glicerina bruta e glicerol nas dietas, aumenta a proporção da AGMI na carne de cordeiros, passando de 46,32\% (0\% de glicerina bruta) até 52,89\% com a inclusão de 21,0\%-30,0\% de glicerina bruta (Tabela 6). AGMI variou de 46,32\% (0\% de glicerol) para 53,1\% com a inclusão de 20,9-24,9\% de glicerol na dieta (Tabela 7). AGS e AGI não são afetados com a inclusão de até $30 \%$ de glicerina bruta e $24,9 \%$ de glicerol na dieta de cordeiros. Pesquisas anteriores constataram que até 24,9\% (Carvalho et al., 2015), 16,8\% (Borghi et al., 2016) e 16,4\% (Rego et al., 2017) de glicerol não prejudicam ou alteram o perfil de ácidos graxos; porém, a qualidade da carne. 
Tabela 6. Porcentagem de ácidos graxos saturados (AGS), insaturados (AGI), monoinsaturado (AGMI) e poli-insaturados (AGPI) e relação n6:n3 do músculo Longíssimus thoracis et lumborum de cordeiros suplementados com diferentes níveis de glicerina bruta

\begin{tabular}{|c|c|c|c|c|c|c|c|c|}
\hline \multirow{2}{*}{ Variáveis } & \multicolumn{4}{|c|}{ Níveis de Glicerina bruta, \% da MS } & \multirow{2}{*}{ SEM } & \multirow{2}{*}{ Gli } & \multirow{2}{*}{$\mathrm{L}$} & \multirow{2}{*}{ Q } \\
\hline & 0 & $2,5-10,00$ & $14,00-20,00$ & $21,00-30,00$ & & & & \\
\hline$\overline{\mathrm{AGS}(\%)}$ & 44,70 & 45,53 & 43,51 & 40,56 & 1,086 & 0,239 & 0,109 & 0,101 \\
\hline AGI $(\%)$ & 54,39 & 53,07 & 55,75 & 59,43 & 1,371 & 0,303 & 0,124 & 0,131 \\
\hline $\operatorname{AGMI}(\%)$ & $46,32^{\mathrm{B}}$ & $43,90^{\mathrm{C}}$ & $48,93^{\mathrm{B}}$ & $52,89^{\mathrm{A}}$ & 1,923 & 0,039 & 0,124 & 0,271 \\
\hline AGPI $(\%)$ & 8,15 & 9,17 & 6,94 & 6,41 & 0,620 & 0,422 & 0,167 & 0,489 \\
\hline n6:n3 & 12,39 & 12,68 & 10,11 & 9,88 & 0,736 & 0,910 & 0,082 & 0,397 \\
\hline
\end{tabular}

$\overline{\mathrm{SEM}}=$ erro quadrado da média. Gli= 0 vs. Glicerina. $\mathrm{L}=$ linear. $\mathrm{Q}=$ quadrática.

Tabela 7. Porcentagem de ácidos graxos saturados (AGS), insaturados (AGI), monoinsaturado (AGMI) e poli-insaturados (AGPI) do músculo Longissimus thoracis et lumborum de cordeiros suplementados com diferentes níveis de glicerol

\begin{tabular}{|c|c|c|c|c|c|c|c|c|}
\hline \multirow{2}{*}{ Variáveis } & \multicolumn{4}{|c|}{ Níveis de Glicerol, \% da MS } & \multirow{2}{*}{ SEM } & \multirow{2}{*}{ Gli } & \multirow{2}{*}{$\mathrm{L}$} & \multirow{2}{*}{ Q } \\
\hline & 0 & $0,98-8,39$ & $10,92-18,68$ & $20,90-24,90$ & & & & \\
\hline$\overline{\mathrm{AGS}(\%)}$ & 44,70 & 45,53 & 42,81 & 40,05 & 1,215 & 0,290 & 0,065 & 0,172 \\
\hline AGI $(\%)$ & 54,39 & 53,07 & 56,54 & 59,70 & 1,447 & 0,346 & 0,083 & 0,187 \\
\hline $\operatorname{AGMI}(\%)$ & $46,32^{\mathrm{B}}$ & $43,90^{\mathrm{C}}$ & $50,00^{\mathrm{A}}$ & $53,10^{\mathrm{A}}$ & 2,025 & 0,050 & 0,093 & 0,345 \\
\hline AGPI (\%) & 8,15 & 9,17 & 6,74 & 6,59 & 0,614 & 0,433 & 0,176 & 0,561 \\
\hline n6:n3 & 12,39 & 12,68 & 10,04 & 9,92 & 0,741 & 0,911 & 0,080 & 0,381 \\
\hline
\end{tabular}

SEM= erro quadrado da média. Gli= 0 vs. Glicerol. L= linear. Q= quadrática.

Os teores de PB, EE, pH e FC da carne não foram influenciados pelos níveis de glicerina bruta na dieta (Tabela 8). A inclusão de até 30\% de glicerina bruta na dieta de cordeiros melhora a concentração de $\mathrm{PB}, \mathrm{EE}, \mathrm{pH}$ e FC da carne. Porém, para PB houve efeito significativo $(\mathrm{P}<0,05)$ com inclusão de até $32,4 \%$ de glicerol na dieta de cordeiros, sendo maior concentração de PB na carne com nível de 21,6\%$32,4 \%$ de glicerol (Tabela 9). Assim, conforme as informações desta metanálise é possível aconselhar o uso de até $30 \%$ de glicerina bruta e 32,4\% de glicerol na dieta de cordeiro, sem provocar efeitos adversos sobre a qualidade nutricional, $\mathrm{pH}$ e força de cisalhamento da carne.

Tabela 8. Porcentagem de proteína bruta (PB \%), extrato etéreo (EE \%), pH final e força de cisalhamento (FC kgf) da carne de cordeiros suplementados com diferentes níveis de glicerina bruta na dieta de acabamento

\begin{tabular}{|c|c|c|c|c|c|c|c|c|}
\hline \multirow[t]{2}{*}{ Variáveis } & \multicolumn{4}{|c|}{ Níveis de Glicerina bruta, \% da MS } & \multirow{2}{*}{ SEM } & \multirow{2}{*}{ Gli } & \multirow{2}{*}{$\mathrm{L}$} & \multirow{2}{*}{ Q } \\
\hline & 0 & $2,5-10$ & $12,00-20,00$ & $21,00-30,00$ & & & & \\
\hline$\overline{\mathrm{PB}(\%)}$ & 20,11 & 19,08 & 19,63 & 21,32 & 0,476 & 0,209 & 0,367 & 0,150 \\
\hline $\mathrm{EE}(\%)$ & 2,80 & 3,74 & 2,59 & 3,38 & 0,264 & 0,324 & 0,906 & 0,994 \\
\hline $\mathrm{pH}$ final & 5,71 & 5,71 & 5,64 & 5,73 & 0,020 & 0,888 & 0,957 & 0,620 \\
\hline $\mathrm{FC}(\mathrm{kgf})$ & 3,86 & 3,91 & 3,84 & 3,61 & 0,068 & 0,959 & 0,113 & 0,054 \\
\hline
\end{tabular}

Gli= 0 vs. Glicerina. $\mathrm{L}=$ Linear. Q= Quadrática

Tabela 9. Porcentagem de proteína bruta (PB \%), extrato etéreo (EE \%), pH final e força de cisalhamento (FC kgf) da carne de cordeiros suplementados com diferentes níveis de glicerol na dieta de acabamento

\begin{tabular}{|c|c|c|c|c|c|c|c|c|}
\hline \multirow[t]{2}{*}{ Variáveis } & \multicolumn{4}{|c|}{ Níveis de Glicerol, \% da MS } & \multirow{2}{*}{ SEM } & \multirow{2}{*}{ Gli } & \multirow{2}{*}{$\mathrm{L}$} & \multirow{2}{*}{ Q } \\
\hline & 0 & $0,98-8,39$ & $10,92-16,78$ & $21,60-32,40$ & & & & \\
\hline$\overline{\mathrm{PB}(\%)}$ & $20,1^{\mathrm{B}}$ & $19,0^{\mathrm{B}}$ & $20,0^{\mathrm{B}}$ & $22,7^{\mathrm{A}}$ & 0,712 & 0,043 & 0,174 & 0,176 \\
\hline $\mathrm{EE}(\%)$ & 2,80 & 3,61 & 3,05 & 2,88 & 0,163 & 0,624 & 0,736 & 0,793 \\
\hline pH final & 5,71 & 5,72 & 5,66 & 5,72 & 0,012 & 0,957 & 0,782 & 0,674 \\
\hline $\mathrm{FC}(\mathrm{kgf})$ & 3,86 & 3,91 & 3,81 & 3,59 & 0,064 & 0,958 & 0,070 & 0,118 \\
\hline
\end{tabular}

Gli= 0 vs. Glicerol. $\mathrm{L}=$ Linear. $\mathrm{Q}=$ Quadrática 


\section{Conclusão}

Níveis de 36,0\% de glicerina bruta ou 32,4\% de glicerol na matéria seca na dieta não comprometem negativamente o desempenho, características da carcaça e qualidade de carne de cordeiros. Neste estudo metanalítico foi possível observar a heterogeneidade na composição de glicerina bruta, sendo que muito poucos estudos trabalharam com pureza igual ou superior a $90 \%$ de glicerol.

\section{Referências bibliográficas}

Almeida, M. T. C., Ezequiel, J. M. B., Paschoaloto, J. R., Perez, H. L., Carvalho, V. B., Castro Filho, E. S. \& van Cleef, E. H. C. B. (2018). Effects of high concentrations of crude glycerin in diets for feedlot lambs: feeding behaviour, growth performance, carcass and non-carcass traits. Animal Production Science, 58(7):1271-1278.

Andrade, G. P., Carvalho, F. F. R., Batista, Â. M. V., Pessoa, R. A. S., Costa, C. A., Cardoso, D. B. \& Maciel, M. V. (2018). Evaluation of crude glycerin as a partial substitute of corn grain in growing diets for lambs. Small Ruminant Research, 16541-47.

ANP, 2018. Agência Nacional do Petróleo, Gás Natural e Biocombustíveis. https://www.biodieselbr.com/noticias/usinas/producao/brasil-produziu-5-35-bilhoes-de-litros-debiodiesel-em-2018-280119. acessado em 26/08/2019.

Avila-Stagno, J., Chaves, A. V., He, M. L., Harstad, O. M., Beauchemin, K. A., McGinn, S. M. \& McAllister, T. A. (2013). Effects of increasing concentrations of glycerol in concentrate diets on nutrient digestibility, methane emissions, growth, fatty acid profiles, and carcass traits of lambs. Journal of Animal Science, 91(2):829-837.

Borghi, T. H., Silva Sobrinho, A. G., Zeola, N. M. B. L., Almeida, F. A., Cirne, L. G. A. \& Lima, A. R. C. (2016). Dietary glycerin does not affect meat quality of Ile de France lambs. Revista Brasileira de Zootecnia, 45(9):554-562.

Carvalho, V. B., Leite, R. F., Almeida, M. T. C., Paschoaloto, J. R., Carvalho, E. B., Lanna, D. P. D. \& Ezequiel, J. M. B. (2015). Carcass characteristics and meat quality of lambs fed high concentrations of crude glycerin in low-starch diets. Meat Science, 110285-292.

Cattelan, A. J. \& Dall'Agnol, A. (2018). The rapid soybean growth in Brazil. Embrapa Soja, 25(1):1-12.

César, A. S., Conejero, M. A., Ribeiro, E. C. B. \& Batalha, M. O. (2019). Competitiveness analysis of "social soybeans" in biodiesel production in Brazil. Renewable Energy, 1331147-1157.

Chanjula, P. (2018). Use of crude glycerin as an energy source for goat diets: a review. Journal of Dairy \& Veterinary Sciences, 21-5.

Chanjula, P., Pakdeechanuan, P. \& Wattanasit, S. (2015). Effects of feeding crude glycerin on feedlot performance and carcass characteristics in finishing goats. Small Ruminant Research, 123(1):95-102.

Chanjula, P., Pongprayoon, S., Kongpan, S. \& Cherdthong, A. (2016). Effects of crude glycerin from waste vegetable oil supplementation on feed intake, ruminal fermentation characteristics, and nitrogen utilization of goats. Tropical Animal Health and Production, 48(5):995-1004.

Costa C. A., Carvalho, F. F R., Guim, A., Andrade, G. P., Cardoso, D. B., Maciel, M. V., Silva, G. G. \& Nascimento, A. G. O. (2019). Carcass characteristics of lambs fed diets with increasing levels of crude glycerin. Asian-Australas Journal Animal Science, DOI: https://doi.org/10.5713/ajas.18.0825

Cruz, O. T. B., Valero, M. V., Zawadzki, F., Rivaroli, D. C., Prado, R. M., Lima, B. S. \& Prado, I. N. (2014). Effect of glycerine and essential oils (Anacardium occidentale and Ricinus communis) on animal performance, feed efficiency and carcass characteristics of crossbred bulls finished in a feedlot system. Italian Journal of Animal Science, 13(4):790-797. doi: http://dx.doi.org/10.4081/ijas.2014.3492.

Cunha, C. M. Cornélio, T. C., Fernandes, A. R. M., Seno, L. O., Ricardo, H. A., Osório, J C. S., Osório, M. T.M., Alves, L. G. C., Oliveira, E. A. (2016). Características da carcaça e qualidade da carne de cordeiros Pantaneiros alimentados com teores crescentes de glicerina bruta. Revista Brasileira de Saúde e Produção Animal, 17(4), p. 729-743. https://doi.org/10.1590/s1519-99402016000400016

D’Aurea E.M.O., Ezequiel, J.M.B., Santos, V.C., D'Aurea, A.P., Carvalho, V.B., Almeida, M.T.C. \& Perez, H.L. (2018). Glicerina na alimentação de cordeiros terminados em confinamento: características 
quantitativas da carcaça e qualitativas da carne. Arquivo Brasileiro de Medicina Veterinária e Zootecnia, 70(6), 1950-1958. DOI: https://doi.org/10.1590/1678-4162-9038

Egea, M., Linares, M. B., Garrido, M. D., Madrid, J. \& Hernández, F. (2016). Feeding Iberian $\times$ Duroc cross pigs with crude glycerine: Effects of diet and gender on carcass and meat quality. Meat Science, 11178-84.

Eiras, C. E., Barbosa, L. P., Marques, J. A., Lima, B. S., Zawadzki, F., Perotto, D. \& Prado, I. N. (2014a). Glycerine levels in the diets of crossbred bulls finished in feedlot: apparent digestibility, feed intake and animal performance. Animal Feed Science and Technology, 197222-226. doi: http://dx.doi.org/10.1016/j.anifeedsci.2014.07.004.

Eiras, C. E., Marques, J. A., Prado, R. M., Valero, M. V., Bonafé, E. G., Zawadzki, F. \& Prado, I. N. (2014b). Glycerin levels in the diets of crossbred bulls finished in feedlot: Carcass characteristics and meat quality. Meat Science, 96(2):930-936. doi: http://dx.doi.org/10.1016/j.meatsci.2013.10.002.

Eiras, C. E., Marques, J. A., Torrecilhas, J. A., Zawadzki, F., Moletta, J. L. \& Prado, I. N. (2013). Glycerin levels in the diets of crossbred bulls finished in feedlot: ingestion behavior, feeding intake and ruminal efficiency. Acta Scientiarum Animal.Sciences, 35(4):411-416. doi: http://dx.doi.org/10.4025/actascianimsci.v35i4.19090.

Françozo, M. C., Prado, I. N., Cecato, U., Valero, M. V., Zawadzki, F., Ribeiro, O. L. \& Visentainer, J. V. (2013). Growth performance, carcass characteristics and meat quality of finishing bulls fed crude glycerin-supplemented diets. Brazilian Archives of Biology and Technology, 56(2):327-336.

Garcia, E. R. M., Murakami, A. E., Ospina-Rojas, I. C. \& Guerra, A. F. Q. G. (2019). Performance, meat quality and fatty acid profile of broiler chickens fed mixed semi-purified glycerin. Animal Production Science, 59(2):295-303.

Gomes, M. A. B., Moraes, G. V., Mataveli, M., Macedo, F. A. F., Carneiro, T. C. \& Rossi, R. M. (2011). Performance and carcass characteristics of lambs fed on diets supplemented with glycerin from biodiesel production. Revista Brasileira de Zootecnia, 40(10):2211-2219.

Gonçalves, L. U., Cerozi, B. S., Silva, T. S. C., Zanon, R. B. \& Cyrino, J. E. P. (2015). Crude glycerin as dietary energy source for Nile tilapia. Aquaculture, 437230-234.

Gunn, P., Neary, M., Lemenager, R. \& Lake, S. (2010a). Effects of crude glycerin on performance and carcass characteristics of finishing wether lambs. Journal of Animal Science, 88(5):1771-1776.

Gunn, P. J., Schultz, A. F., Van Emon, M. L., Neary, M. K., Lemenager, R. P., Rusk, C. P. \& Lake, S. L. (2010b). Effects of elevated crude glycerin concentrations on feedlot performance, carcass characteristics, and serum metabolite and hormone concentrations in finishing ewe and wether lambs. The Professional Animal Scientist, 26(3):298-306.

Lage, J. F., Paulino, P. V. R., Pereira, L. G. R., Duarte, M. S., Valadares Filho, S. C., Oliveira, A. S. \& Lima, J. C. M. (2014). Carcass characteristics of feedlot lambs fed crude glycerin contaminated with high concentrations of crude fat. Meat Science, 96(1):108-113. doi: http://dx.doi.org/10.1016/j.meatsci.2013.06.020

Lage, J. F., Paulino, P. V. R., Pereira, L. G. R., Valadares Filho, S. C., Oliveira, A. S., Detmann, E. \& Lima, J. C. M. (2010). Glicerina bruta na dieta de cordeiros terminados em confinamento. Pesquisa Agropecuária Brasileira, 45(9):1012-1020.

Ma, F. \& Hanna, M. A. (1999). Biodiesel production: a review. Bioresource Technology, 70(1):1-15.

Merlim F.A., da Silva Sobrinho, A.G., Borghi, T.H., Zeola, N.M.B.L., Cirne, L.G.A., Romanzini, E.P., Andrade, N. \& Almeida, F.A. (2015). Ile De France Lambs Fed Diets Containing Glycerin: Characteristics of Carcass and Commercial Cuts. Journal of Agricultural Engineering and Biotechnology, 3(2), 79.83. doi https://doi.org/10.18005/jaeb0302005

Nobrega, M. M., Olivato, J. B., Bilck, A. P., Grossmann, M. V. E. \& Yamashita, F. (2012). Glycerol with different purity grades derived from biodiesel: Effect on the mechanical and viscoelastic properties of biodegradable strands and films. Materials Science and Engineering: C, 32(8):2220-2222. doi: http://dx.doi.org/10.1016/j.msec.2012.06.005

Orrico Jr, M. A. P., Bottini Filho F. D. E., de Vargas Junior, F. M., Orrico, A. C. A. \& Osório, J. C. S. (2015). Crude glycerin in the diets of confined lambs: performance, carcass traits and economic feasibilityBioscience. Journal, 31, (4), 1152-1158. doi: https://doi.org/10.14393/bj-v31n4a2015-26226 
Pellegrin A.C. R. S., Pires, C. C., Carvalho, S., Pacheco, P. S., Pelegrini, L. F. V. Griebler, L. \& Venturini, R. S. (2012). Glicerina bruta no suplemento para cordeiros lactentes em pastejo de azevém. Ciência Rural, 42(8), 1477-1482. doi: https://doi.org/10.1590/s0103-84782012005000052

Pellegrin, A. C. R. S., Pires, C.C., Mello, R.O., Müller, L., Carvalho, S. \& Lopes, J.F. (2013). Glicerina bruta no suplemento e seus efeitos nas características da carcaça e nos componentes do peso vivo de cordeiros lactentes. Arquivo Brasileiro de Medicina Veterinária e Zootecnia, 65(5), 1509-1518. doi: https://doi.org/10.1590/s0102-09352013000500032

Prado, I. N., Cruz, O. T. B., Valero, M. V., Zawadzki, F., Eiras, C. E., Rivaroli, D. C. \& Visentainer, J. V. (2016). Effects of glycerin and essential oils (Anacardium occidentale and Ricinus communis) on the meat quality of crossbred bulls finished in a feedlot. Animal Production Science, 55(12):2105-2114. doi: http://dx.doi.org/10.1071/AN14661.

Quispe, C. A. G., Coronado, C. J. R. \& Carvalho Jr, J. A. (2013). Glycerol: Production, consumption, prices, characterization and new trends in combustion. Renewable and Sustainable Energy Reviews, 27(0):475493. doi: http://dx.doi.org/10.1016/j.rser.2013.06.017

Rego, F. C. A., Françozo, M. C., Ludovico, A., Castro, F. A. B., Zundt, M., Lupo, C. R. \& Castilho, C. (2017). Fatty acid profile and lambs' meat quality fed with different levels of crude glycerin replacing corn. Semina: Ciências Agrárias, 38(4):2051-2064.

Rezende, L. C., Heimbach, N. d. S., Ítavo, C. C. B. F., Ítavo, L. C. V., Morais, M. d. G., Brumatti, R. C. \& Silva, J. A. (2017). Intake, feeding behaviour, digestibility, performance, carcass characteristics and meat quality of lambs fed different levels of semi-purified glycerine in the diet. Archives of Animal Nutrition, 71(6):470-485.

Romanzini, E. P., Silva Sobrinho, A. G., Valença, R. L., Borghi, T. H., Andrade, N. \& Bernardes, P. A. (2018). Feedlot of lambs fed biodiesel co-products: performance, commercial cuts and economic evaluation. Tropical Animal Health and Production, 50(1), 155-160. DOI: https://doi.org/10.1007/s11250-017-1416-3

Saleem, A. M. \& Singer, A. M. (2018). Growth performance and digestion of growing lambs fed diets supplemented with glycerol. Animal, 12(5):959-963.

Schröder, A. \& Südekum, K. H. (1999). Glycerol as a by-product of biodiesel production in diets for ruminants. Paper presented at the Wratten, N., Salisbury, PA (Eds.), New Horizons for an Old Crop. Proc. 10th Int. Rapeseed Congr., Canberra, Australia. Paper no. 241. The Regional Institute Ltd., Gosford, New South Wales, Australia, Canberra.

Silva, L. G., Torrecilhas, J. A., Ornaghi, M. G., Eiras, C. E., Prado, R. M. \& Prado, I. N. (2014). Glycerin and essential oils in the diet of Nellore bulls finished in feedlot: animal performance and apparent digestibility. Acta Scientiarum Animal.Sciences, 36(2):177-184. doi: http://dx.doi.org/10.4025/actascianimsci.v36i2.23089.

Silva, R. R., Facuri, L. M. A. M., Carvalho, G. G. P., Silva, F. F., Siminonaro, J. I., Sampaio, C. B. \& Araújo, M. L. G. M. L. (2017). Meat quality of heifers finished on pasture with tropical and supplemented with glycerin. Ciencias e Investigación Agraria, 44(3):207-214. doi: http:/dx.doi.org/10.7764/rcia.v44i3.1861.

Souza, L. L., Azevêdo, J. A. G., Araújo, G. G. L., Santos-Cruz, C. L. \& Oliveira, B. S. (2015). Crude glycerin for Santa Inês and F1 Dorper $\times$ Santa Inês lambs. Small Ruminant Research, 129, 1-5. DOI: https://doi.org/10.1016/j.smallrumres.2015.06.006

Terré, M., Nudda, A., Casado, P. \& Bach, A. (2011). The use of glycerine in rations for light lamb during the fattening period. Animal Feed Science and Technology, 164(3):262-267.

USDA - United States Department of Agriculture. (2019). Oilseeds: World Markests and Trade. Washington DC. 22/0602019. Disponível: https://apps.fas.usda.gov/psdonline/circulars/oilseeds.pdf

Vieira \& Silva, et al. (2018)

Recebido: 28 de agosto, 2019.

Aprovado: 1 de novembro, 2019.

Publicado: 5 de dezembro, 2019.

Licenciamento: Este artigo é publicado na modalidade Acesso Aberto sob a licença Creative Commons Atribuição 4.0 (CC-BY 4.0), a qual permite uso irrestrito, distribuição, reprodução em qualquer meio, desde que o autor e a fonte sejam devidamente credita 